\title{
Subacute Bowel Obstruction Secondary to an Obturator Hernia
}

\author{
Long-Zhi Zheng, Chang-Yue Zheng, Bin Zu and Wei Lin \\ Department of Gastrointestinal Surgery, The Affiliated Hospital of Putian University, Putian, Fujian Province, China
}

\begin{abstract}
Obturator hernia is a rare abdominal wall hernia and is one of the rare causes of intestinal obstruction. Due to lack of specific clinical symptoms in the early stage, and the deep position of hernia absence of surface swelling, few cases are diagnosed definitively before surgery. Therefore, obturator hernia is still a serious disease in clinical practice, with high mortality and morbidity. Herein, we report an 85-year female who was hospitalised with bowel obstruction. Preoperative computed tomography scan was performed, which revealed an incarcerated obturator hernia preoperatively; and it was treated successfully. This report reviews the diagnosis and treatment of obturator hernia by describing its anatomy, clinical manifestations and clinical signs. Increased awareness and proper management of the disease will lead to a decline in mortality and morbidity.
\end{abstract}

Key Words: Obturator hernia, Intestinal obstruction, Howship-Romberg sign.

How to cite this article: Zheng LZ, Zheng CY, Zu B, Lin W. Subacute bowel obstruction secondary to an obturator hernia. $J$ Coll Physicians Surg Pak 2020; 30(2):210-212.

\section{INTRODUCTION}

Obturator hernia is not a common clinical condition and accounts for less than one percent of all intra-abdominal hernias. ${ }^{1}$ It often occurs in elderly, multiparous female patients with debilitating conditions (e.g., chronic obstructive pulmonary disease, diabetes, chronic constipation, sleep disorders, malnutrition, living alone, and low educational level), which earned it the nickname, "little old lady's hernia".2 A lack of typical clinical manifestations of obturator hernia often leads to delayed surgery, resulting in high morbidity and mortality $(13 \%$ to $40 \%)^{3}$ Preoperative computerised axial tomography scanning (CAT/CT scan) can improve the diagnosis of obturator hernia. Accurate diagnosis and timely operation are the key to improve the prognosis of patients with obturator hernia.

\section{CASE REPORT}

A 85-year woman was admitted to the emergency department of the Affiliated Hospital on May 6, 2017, because of abdominal pain of four days' duration. The patient had no obvious cause of periumbilical pain. The pain was paroxysmal, which appeared once half an hour, lasted for more than 10 minutes, and was accompanied by nausea, vomiting, abdominal distension, and constipation; but no feeling of cold and fever. The

Correspondence to: Wei Lin, Department of Gastrointestinal Surgery, The Affiliated Hospital of Putian University, No. 999, Dongzheng Road, Licheng District, Putian 351100, Fujian

Province, China

E-mail:linwbj@outlook.com

Received: March 12, 2019; Revised: July 25, 2019;

Accepted: August 06, 2019 vomiting contained bile and stomach contents. The patient lived alone and gave history of occasional abdominal pain and discomfort in the past, which got relieved by oral medicines to help digestion, so she did not visit any hospital for treatment. In addition, she did not have any past history of abdominal surgery. She was a tall, thin person, and in delicate health (BMI 14.22 $\mathrm{kg} / \mathrm{m}^{2}$ ). The abdomen was not tender but it was slightly distended. She also complained of pain in the periumbilical area. The bowel sounds were hyperactive which suggested obstruction. No external hernia could be detected and rectal examination was unremarkable. Her haemoglobin concentration was $10.5 \mathrm{~g} / \mathrm{dl}$, peripheral white blood count $14.49 \times 10^{2} / \mathrm{L}$, neutrophilis $91.60 \%$, platelet count $157 \times 109 / \mathrm{L}$, plasma albumin was $33.7 \mathrm{~g} / \mathrm{L}$, plasma sodium $121.3 \mathrm{mmol} / \mathrm{L}$, and plasma chloridie $88.4 \mathrm{mmol} / \mathrm{L}$. CT scan suggested a right obturator hernia before operation (Figure 1). Preoperative diagnosis included acute intestinal obstruction, right obturator hernia, hypoalbuminemia, and electrolyte disturbances.

As the patient was not in a good condition, we wanted to complete the surgery quickly so the abdomen was opened by a lower midline incision after an informed consent. There was a small amount of yellowish exudative fluid in the pelvic cavity, with the antimesenteric border of the ileum incarcerated into the right obturator foramen nearly $100 \mathrm{~cm}$ from the ileoceacal junction, resulting in an obturator hernia (Richter's hernia) (Figure 2). Using the index finger to separate the adhesions around the foramen and dilating the obturator foramen with a vessel forceps, the knuckle of intestine was withdrawn from the foramen by pulling it gently. The incarcerated small intestine accounted for about $3 / 4$ of the circumference of the intestine and did not involve mesenteric 


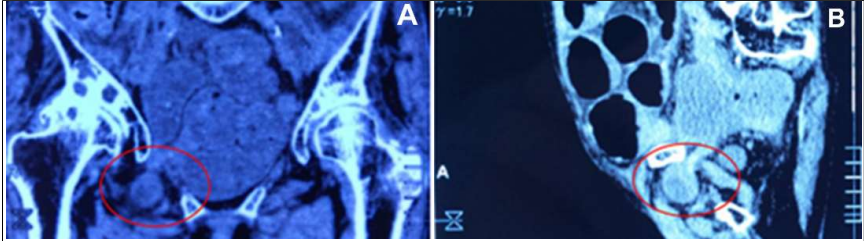

Figure 1: Computed tomography images of the pelvis showing the obturator hernia on the right side: $(A)$ Sagittal section, and (B) Coronal section. There is a fluid-filled loop of small bowel herniating through the right obturator foramen (circles).
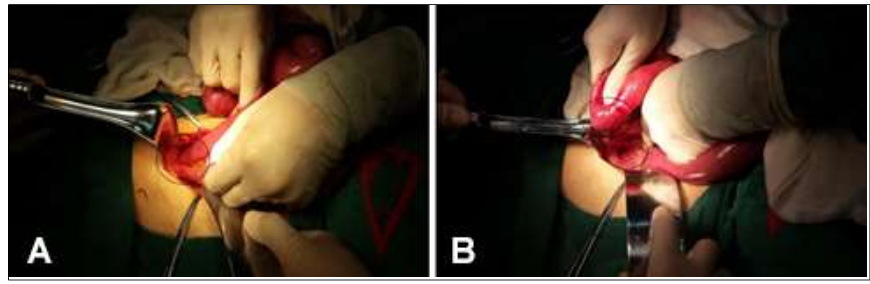

Figure 2: Intraoperative images of the right sided obturator hernia: (A) Herniation of ileal loops through the right obturator canal; (B) Hernial sac and incarcerated bowel (Richter type hernia) seen after reduction (circles).

vessels. This incarcerated bowel was a bit black, but after being warmed with hot isotonic saline gauze for about 10 minutes, the intestinal wall gradually turned red, and peristalsis was normal. As the mesenteric arterial pulse was normal, so it was put back into the abdominal cavity. The obturator hernia defect was relatively small, which could only accommodate the tip of the little finger, and the general condition of the patient was not good. The obturator canal opening was closed with No. 4 non-absorbable sutures. The patient recovered well and was discharged from hospital on the seventh postoperative day.

She was fine on follow-up six months after surgery and regained some weight.

\section{DISCUSSION}

An obturator hernia is defined as one which passes between the superior pubic ramus of the pubic bone and the fibrous obturator membrane. The obturator canal, which is $2-3 \mathrm{~cm}$ long and $1 \mathrm{~cm}$ wide, contains the obturator vein, artery, and nerve branches. Strangulated obturator hernia is a rare condition first described by Arnaud de Ronsil in France in 1724,4 and was first successfully repaired by Henry Obre in 1851.5 Obturator hernia is uncommon and account for only 0.05 to $0.70 \%$ of all hernias and only 0.2 percent of all mechanical small bowel obstructions needing surgical treatment.1 The incidence of the right obturator hernia is two times that of the left side, which may be related to the distribution of the sigmoid colon covering the left obturator orifice. 6 Females are affected at least six times more frequently than males. ${ }^{3}$ Most patients belong to seventh and eighth decades of life. It is often found in elderly women who are wasted and multiparous, because of a wider pelvis, larger triangular obturator canal and greater transverse diameter. Other risk factors include chronic obstructive pulmonary disease, chronic constipation, ascites and kyphosis, etc. The mechanism may be increased intra-abdominal pressure. Emaciation may also be an important factor. It is postulated that with severe weight loss, there is a decrease in the protective preperitoneal fat from the obturator canal. The BMI of these patients is between 13-19.7

There are four cardinal features of the obturator hernia: (1) the Howship-Romberg sign, which is pain along the obturator nerve in the thigh caused by compression of the obturator nerve by the hernial sac. The pain is exacerbated by extension, abduction, or inward rotation of the thigh. (2) intestinal obstruction, (3) repeated episodes of bowel obstruction that resolve spontaneously, and (4) a palpable mass in the groin with flexion of the thigh, abduction, extension, and medial rotation. The HowshipRomberg sign and intestinal obstruction are the most common presentations. Although the Howship-Romberg sign is pathognomonic of obturator hernia, the positive rate is only $15 \%-50 \% .8$ The Howship-Romberg sign was negative in our patient. The contents of the obturator hernia are mainly small intestine, and sometimes include caecum and uterus, appendix, bladder, omentum, fallopian tube, and ovary. ${ }^{9}$

Non-specific signs and symptoms make the preoperative diagnosis of an obturator hernia difficult, which was related to the high mortality, reported to be as high as $40 \% .^{3}$ The preoperative diagnostic methods of obturator hernia include: abdominal X-ray, B-ultrasound, CT, etc. CT is the gold standard for preoperative diagnosis of obturator hernia. ${ }^{10}$ Obturator hernia is prone to incarceration, intestinal necrosis and perforation, and manual reduction is more dangerous than in other types of hernia. Therefore, once the intestinal obstruction caused by obturator hernia is diagnosed or suspected, surgical treatment should be selected.

Operative repair of obturator hernia may be performed by laparotomy approach, laparoscopic approach, or obturator and inguinal approaches. The technique used to repair the defect has varied from a simple suture closure or biological tissue closure (such as rib cartilage, peritoneum) to polypropylene mesh placement, some of which are ingenious but equally time-consuming.

In summary, early diagnosis and treatment of this rare hernia are essential for good outcome. A lower midline abdominal incision provides excellent exposure of the constricting ring from above, and it can be opened and closed quickly, which is especially suitable for patients who need partial bowel resection. In sick elderly patients, when speed is important, this incision has advantages over other approaches, which have been described. 


\section{PATIENT'S CONSENT:}

Informed consent was obtained from the patient for publication of this case report and any accompanying images.

\section{CONFLICT OF INTEREST:}

Authors declared no conflict of interest.

\section{AUTHORS' CONTRIBUTION:}

WL: Corresponding author, the consultant surgeon who run the operations.

LZZ: Contribution by giving the study concept and analysis as well as wrote the paper.

CYZ: Literature review as well as assistant surgeon in the patient's operations.

BZ: Data collection and image processing.

\section{FUNDING:}

This study was sponsored by the Natural Science Foundation of Fujian Province (No. 2019J01582) and the Science and Technology Foundation of the Education Department of Fujian Province (No. JAT170520).

\section{REFERENCES}

1 Gürlich R. Lindner J. Obturator hernia. Rozhl Chir 1996; 75: 307-9.
2 Kordzadeh A, Charalabopoulos A. The little old lady's hernia. Surgery 2018; 163:482.

3 Mantoo SK, Mak K, Tan TJ. Obturator hernia: Diagnosis and treatment in the modern era. Singapore Med J 2009; 50: 866-70.

4 Green BT. Strangulated obturator hernia: Still deadly. South Med J 2001; 94:81-83.

5 Skandalakis LJ, Androulakis J, Colborn GL, Skandalakis JE. Obturator hernia: Embryology, anatomy, and surgical applications. Surg Clin North Am 2000; 80:71-84.

6 Igari K, Ochiai T, Aihara A, Kumagai Y, lida M, Yamazaki S. Clinical presentation of obturator hernia and review of the literature. Hernia 2010; 14:409-13.

7 Nakayama T, Kobayashi S, Shiraishi K, Nishiumi T, Mori S, Isobe $\mathrm{K}$, et al. Diagnosis and treatment of obturator hernia. Keio J Med 2002; 51:129-32.

8 Mitchell A, Morera DS, Holsten S. Surgical management of an obturator hernia. Am Surg 2018; 84:e403-4.

9 Sá NC, Silva VCM, Carreiro PRL, Matos Filho AS, Lombardi IA. Rare case of incarcerated obturator hernia: Case report and review of literature. Int J Surg Case Rep 2017; 37:157-60.

10 Light D, Razi K, Horgan L. Computed tomography in the investigation and management of obturator hernia. Scott Med J 2016; 61:103-5. 